\title{
On hierarchies and communication
}

\author{
René van den Brink
}

Received: 10 September 2010 / Accepted: 14 May 2011 / Published online: 23 June 2011

(C) The Author(s) 2011. This article is published with open access at Springerlink.com

\begin{abstract}
Many economic and political organizations have some relational structure, meaning that participating agents do not only differ with respect to certain individual characteristics such as wealth and preferences, but also belong to some relational structure in which they usually take different positions. Two examples of such structures are communication networks and hierarchies. In the literature, the distinction between these two types of relational structures is not always clear. In models of restricted cooperation, this distinction should be defined by properties of the set of feasible coalitions. We characterize the sets of feasible coalitions in communication networks and compare them with sets of feasible coalitions arising from hierarchies.
\end{abstract}

\section{Introduction}

Many economic and political organizations have some relational structure, meaning that participating agents do not only differ with respect to certain individual characteristics such as wealth and preferences, but also belong to some relational structure in which they usually take different positions. Two examples of such structures are communication networks and hierarchies. Whereas hierarchical relations are usually between different types of agents (or agents having different roles) and thus are asymmetric relations, communication relations might be symmetric (and between similar type of agents) or asymmetric. Bala and Goyal (2000), for example, consider communication relations between symmetric agents and a communication link between two agents means that these two agents share their information with one another (the two-sided case) or only the agent who builds the relation gets access to the

R. van den Brink ( $\varangle)$

Department of Econometrics and Tinbergen Institute, VU University,

De Boelelaan 1105, 1081 HV Amsterdam, The Netherlands

e-mail: jrbrink@feweb.vu.nl 
information of the other (the one-sided case). In their model of network formation agents can unilaterally decide to build or delete communication links. In the network formation model of Jackson and Wolinsky (1996), agents can unilaterally decide to delete links, but for building links mutual agreement is needed. Their model applies the static model of restricted communication in cooperative games of Myerson (1977) where connectedness determines the possibilities of cooperation. An example of asymmetric communication relations is given by Dewatripont and Tirole (2005) who consider communication as a "transfer of knowledge" between a sender and a receiver. They formulate a principal-agent model to communication as a moral hazard problem between the sender and receiver.

Although these are different models of communication, their seems to be consensus in the sense that for each form of communication the underlying idea is "connectedness": agents must be connected in order to share information or to be able to cooperate. There is less consensus about the meaning of hierarchy. It is even not clear whether hierarchy implies authority or not, as expressed by Hart and Moore (2005). But even when agreeing that hierarchy is about authority, the implications of authority differ across different models. Moreover, there can be different forms of authority within one organization, see Aghion and Tirole (1997) who distinguish between "formal authority" (the right to decide) and "real authority" (the effective control over decisions).

Few attempts are made to build a consistent theory on organizations combining both communication and hierarchies in the relational structure. In the field of restricted cooperation, an attempt is made by Demange (2004), but as acknowledged implicitly in that article this is mainly about communication. The so-called hierarchical outcomes that are defined in that article are extreme points of the Core of the restricted game introduced in Myerson (1977) for communication graph games. In that restricted game, only coalitions that are connected in a communication network are feasible and can cooperate. Le Breton et al. (1992) and Demange (1994, 2004), consider a modified Core concept where coalitional stability is required only for these feasible coalitions. Therefore, only for connected coalitions, it is required that the sum of payoffs of the players is at least the worth of that coalition. They show that this set of Core-stable payoff vectors is nonempty if the game is superadditive and the communication graph is cycle-free, respectively a tree. This is interesting since superadditivity of a TU-game does not guarantee the existence of a Core-stable payoff vector.

A model that studies restrictions in cooperation arising from hierarchies is that of a game with a permission structure. In those games, it is assumed that players who participate in a cooperative TU-game are part of a hierarchical organization in which there are players that need permission or approval from certain other players before they are allowed to cooperate. Two approaches to games with a permission structure are considered. In the conjunctive approach as developed in Gilles et al. (1992) and van den Brink and Gilles (1996), it is assumed that each player needs permission from all its predecessors before it is allowed to cooperate with other players. This implies that a coalition is feasible if and only if for every player in the coalition it holds that all its predecessors belong to the coalition. Alternatively, in the disjunctive approach as developed in Gilles and Owen (1994) and van den Brink (1997), it is assumed that each player (except the top-players) needs permission from at least one of its predecessors before it is allowed to cooperate. Consequently, a coalition 
is feasible if and only if every player in the coalition (except the top-players) has at least one predecessor who also belongs to the coalition. In Algaba et al. (2004), it is shown that the sets of feasible coalitions arising from these permission structures are antimatroids being a well-known combinatorial structure representing hierarchies, see Dilworth (1940) and Edelman and Jamison (1985). A set of feasible coalitions is an antimatroid if it contains the empty set and satisfies accessibility (meaning that every nonempty feasible coalition has at least one player that can leave the coalition leaving behind a feasible subcoalition) and is closed under union (meaning that the union of two feasible coalitions is also feasible).

In the field of restricted cooperation, the difference between hierarchies and communication networks should be defined by properties of the sets of feasible coalitions. The purpose of the underlying article is to make clear this distinction. The main result is a characterization of the sets of feasible coalitions arising from communication networks (called communication feasible sets) using properties that are similar to those that define antimatroids. It turns out that the communication feasible sets are characterized by a weaker union property, but a stronger accessibility property. The weaker union property is union stability meaning that the union of two feasible coalitions that have a nonempty intersection is also feasible (see, Algaba et al. (2001)). The stronger accessibility property is 2-accessibility meaning that every feasible coalition with two or more players has at least two players that can leave the coalition leaving behind a feasible coalition.

After this characterization of communication feasible sets for arbitrary communication graphs, we characterize the communication feasible sets arising from special communication graphs that gained attention in the economic literature. For example, adding closedness under intersection (meaning that the intersection of two feasible coalitions is also feasible) yields communication feasible sets arising from cyclecomplete communication graphs. Further, we consider other special graphs such as cycle-free communication graphs, line-graphs, and trees.

The article is organized as follows. In Sect. 2, we review cooperation restrictions arising from limited communication and hierarchical restrictions represented by antimatroids and games with a permission structure. In Sect. 3, we give a full characterization of communication feasible sets using properties that are similar to those that define an antimatroid. In Sect. 4, we consider some special classes of communication networks. Finally, Sect. 5 contains some concluding remarks.

\section{Communication and hierarchies in cooperative games}

A situation in which a finite set of players can obtain certain payoffs by cooperation can be described by a cooperative game with transferable utility, or simply a TU-game, being a pair $(N, v)$, where $N \subseteq \mathbb{N}$ is a finite set of players and $v: 2^{N} \rightarrow \mathbb{R}$ is a characteristic function on $N$ satisfying $v(\emptyset)=0$. For any coalition $S \subseteq N, v(S)$ is the worth of coalition $S$, meaning that the members of coalition $S$ can obtain a total payoff of $v(S)$ by agreeing to cooperate. A TU-game $(N, v)$ is superadditive if $v(S \cup T) \geq v(S)+v(T)$ for all $S, T \subseteq N$ with $S \cap T=\emptyset$, i.e., when two disjoint coalitions merge in a superadditive game then it is always possible to reallocate the 
worth of these coalitions in such a way that all players in the coalition benefit from the merger.

In a superadditive TU-game, one might expect that eventually the "grand coalition" $N$ will form. Main question then is to determine the distribution of payoffs over individual players. A payoff vector of an $n$-player TU-game $(N, v)$ is an $n$-dimensional vector $x \in \mathbb{R}^{n}$ giving a payoff $x_{i} \in \mathbb{R}$ to any player $i \in N$. A solution for TU-games is a mapping $F$ that assigns to every game $(N, v)$ a set of payoff vectors $F(N, v) \subseteq \mathbb{R}^{n}$. A famous and widely applied solution is the Core which assigns to every game the set of efficient and coalitionally stable payoff vectors, i.e., $\operatorname{Core}(N, v)=\left\{x \in \mathbb{R}^{n} \mid \sum_{i \in N} x_{i}=v(N)\right.$ and $\sum_{i \in S} x_{i} \geq v(S)$ for all $\left.S \subset N\right\}$. For general TU-games, a Core payoff vector need not exist, even not for superadditive games.

In a TU-game, any subset $S \subseteq N$ is assumed to be able to form a coalition and earn the worth $v(S)$. However, in most economic and political organizations not every set of participants can form a feasible coalition. For example, for two players to cooperate it might be necessary that they know each other, or it might be sufficient that there are other players in the coalition that know both players, or there are players that know these players, and so on. In hierarchical organizations it might be that players need approval from their superiors in order to cooperate within a coalition. Therefore, in cooperative game theory models have been developed in which there are restrictions on coalition formation. If there are restrictions on coalition formation then the set of feasible coalitions $\mathcal{F} \subseteq 2^{N}$ need not contain all subsets of the player set $N$. It is obvious that considering the Core stability inequalities only for a subset of coalitions yields a set of stable payoff vectors that contains the Core of the unrestricted game, and may even yield nonemptiness while the unrestricted game has an empty Core.

\subsection{Communication}

One of the most well-known restrictions on coalition formation are communication restrictions as introduced in Myerson (1977). In this model, there is a communication network on the set of players in a cooperative game and a coalition $S$ is feasible if and only if the players in $S$ are connected within this communication network. This communication network is represented by an undirected graph on the set of players.

An undirected graph is a pair $(N, G)$ where $N$ is the set of nodes and $G \subseteq$ $\{\{i, j\} \mid i, j \in N, i \neq j\}$ is a collection of subsets of $N$ such that each element of $G$ contains precisely two elements. The elements of $G$ represent bilateral communication links and are referred to as edges or links. Since the nodes in a graph represent the positions of players in a communication network we refer to the nodes as players. If there is a link between two players then we call them neighbors and we denote the set of neighbors of player $i$ by $R_{G}(i)=\{j \in N \mid\{i, j\} \in G\}$. A sequence of $k$ different players $\left(i_{1}, \ldots, i_{k}\right)$ is a path in $(N, G)$ if $\left\{i_{h}, i_{h+1}\right\} \in G$ for $h=1, \ldots, k-1$. Two distinct players $i$ and $j, i \neq j$, are connected in graph $(N, G)$ if there is a path $\left(i_{1}, \ldots, i_{k}\right)$ with $i_{1}=i$ and $i_{k}=j$. A coalition $S \subseteq N$ is connected in graph $(N, G)$ if every pair of players in $S$ is connected by a path that only contains players from $S$, i.e., for every $i, j \in S, i \neq j$, there is a path $\left(i_{1}, \ldots, i_{k}\right)$ such that $i_{1}=i, i_{k}=j$, and 


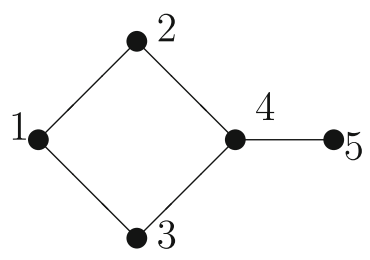

Fig. 1 Communication graph $(N, G)$ of Example 1

$\left\{i_{1}, \ldots, i_{k}\right\} \subseteq S$. A maximally connected subset of coalition $S$ in $(N, G)$ is called a component of $S$ in that graph, i.e., $T \subseteq S$ is a component of $S$ in $(N, G)$ if and only if (i) $T$ is connected in $(N, G(S))$ and (ii) for every $h \in S \backslash T$ the coalition $T \cup\{h\}$ is not connected in $(N, G(S))$, where $G(S)=\{\{i, j\} \in G \mid\{i, j\} \subseteq S\}$ is the set of links between players in $S$.

A sequence of players $\left(i_{1}, \ldots, i_{k}, i_{1}\right)$ is a cycle in $(N, G)$ if $\left(i_{1}, \ldots, i_{k}\right)$ is a path in $(N, G)$ and $\left\{i_{k}, i_{1}\right\} \in G$. A graph $(N, G)$ is cycle-free when it does not contain any cycle. A player $i \in N$ is called a pending player if it is connected to exactly one other player, i.e., if $|\{g \in G \mid i \in g\}|=1$. Note that a cycle-free communication graph $(N, G)$ with $|N| \geq 2$ and $G \neq \varnothing$ has at least two pending players. A graph that is connected and cycle-free is called a tree.

Example 1 Consider the communication graph $(N, G)$ on $N=\{1, \ldots, 5\}$ given by $G=\{\{1,2\},\{1,3\},\{2,4\},\{3,4\},\{4,5\}\}$, see Fig. 1. Players 1 and 5 are connected by two paths: $(1,2,4,5)$ and $(1,3,4,5)$. Coalition $\{1,4,5\}$ has two components: $\{1\}$ and $\{4,5\}$. This communication graph has a cycle $(1,2,4,3,1)$, and player 5 is the only pending player.

A triple $(N, v, G)$ with $(N, v)$ a TU-game and $(N, G)$ an undirected communication graph is called a communication graph game. In the communication graph game $(N, v, G)$ players can cooperate if and only if they are able to communicate with each other, i.e., a coalition $S$ is feasible if and only if it is connected in $(N, G)$. In other words, the set of feasible coalitions in a communication graph game $(N, v, G)$ is the set of coalitions $\mathcal{F}_{G} \subseteq 2^{N}$ given by

$$
\mathcal{F}_{G}=\{S \subseteq N \mid S \text { is connected in }(N, G)\} .
$$

We refer to this set as the communication feasible set of communication graph $(N, G)$. Myerson (1977) introduced the restricted game of a communication graph game $(N, v, G)$ as the TU-game $\left(N, v_{G}\right)$ in which every feasible coalition $S$ can earn its worth $v(S)$. Whenever $S$ is not feasible it can earn the sum of the worths of its components in $(N, G)$. Denoting the set of components of $S \subseteq N$ in $(N, G)$ by $C_{G}(S)$, the restricted game $\left(N, v_{G}\right)$ corresponding to communication graph game $(N, v, G)$ thus is given by ${ }^{1}$

$$
v_{G}(S)=\sum_{T \in C_{G}(S)} v(T) \text { for all } S \subseteq N .
$$

\footnotetext{
1 Note that $C_{G}(S)$ is a partition of $S$.
} 
As solution Myerson (1977) proposes to take for every communication graph game the Shapley value (Shapley (1953)) of the corresponding restricted game, a solution that is later named the Myerson value for communication graph games. Alternatively, Le Breton et al. (1992) and Demange (1994, 2004), consider a restricted Core concept where coalitional stability is required only for feasible coalitions, i.e., they consider the solution $C(N, v, G)=\left\{x \in \mathbb{R}^{n} \mid \sum_{i \in T} x_{i}=v(T)\right.$ for all $T \in C_{G}(N)$, and $\sum_{i \in S} x_{i} \geq v(S)$ for all $S \in \mathcal{F}_{G}$. They show that this set of Core payoff vectors is nonempty if the game is superadditive and the communication graph is cycle-free ${ }^{2}$ respectively a tree. ${ }^{3}$ This is interesting since, as mentioned before, superadditivity of a game $v$ does not guarantee the existence of a Core-stable payoff vector for $v$.

\subsection{Hierarchies}

A model that studies restrictions in coalition formation arising from hierarchies is that of a game with a permission structure. In those games, it is assumed that players who participate in a cooperative TU-game are part of a hierarchical organization in which there are players that need permission or approval from certain other players before they are allowed to cooperate. For a finite set of players $N$ such a hierarchical organization is represented by an irreflexive directed graph $(N, D)$ with $D \subseteq N \times N$ such that $(i, i) \notin D$ for all $i \in N$, referred to as a permission structure on $N$. The directed links $(i, j) \in D$ are called $\operatorname{arcs}$. The players in $F_{D}(i):=\{j \in N \mid(i, j) \in D\}$ are called the successors or followers of player $i$, while the players in $P_{D}(i):=\{j \in N \mid(j, i) \in D\}$ are called the predecessors of $i$. A sequence of different players $\left(i_{1}, \ldots, i_{k}\right)$ is a directed path between players $i$ and $j, i \neq j$, in a permission structure $(N, D)$ if $i_{1}=i, i_{k}=j$, and $\left(i_{h}, i_{h+1}\right) \in D$ for all $1 \leq h \leq k-1$. It is widely accepted that many hierarchies in economic organizations are acyclic. Therefore, we only consider acyclic permission structures, i.e., we assume that there exists no directed path $\left(i_{1}, \ldots, i_{k}\right)$ with $\left(i_{k}, i_{1}\right) \in D$. Note that in an acyclic permission structure there can be two directed paths from player $i$ to player $j \neq i$. Also note that in an acyclic permission structure $D$ there always exists at least one player with no predecessors, i.e., $T O P(D):=\left\{i \in N \mid P_{D}(i)=\emptyset\right\} \neq \emptyset$. We refer to these players as the top-players in the permission structure. If there is a unique top-player, and there is exactly one directed path from the top-player to any other player then the permission structure is a directed or rooted tree.

Two approaches to games with a permission structure are considered. In the conjunctive approach as developed in Gilles et al. (1992) and van den Brink and Gilles (1996), it is assumed that each player needs permission from all its predecessors before it is allowed to cooperate. This implies that a coalition $S \subseteq N$ is feasible if and only if for every player in the coalition it holds that all its predecessors belong to the coalition.

\footnotetext{
2 Under these conditions, this solution coincides with the (unrestricted) Core of the restricted game $\left(N, v_{G}\right)$, see also Kaneko and Wooders (1982). For games that are not superadditive another main question is which coalitions one might expect to form. Demange $(1994,2004)$ generalizes solutions to these cases by allowing the players to eventually form a partition of the player set.

3 Demange (2004) also shows that in case the communication graph contains a cycle, one can always find a superadditive game such that the corresponding set of Core payoff vectors is empty.
} 


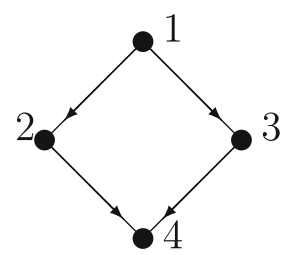

Fig. 2 Permission structure $(N, D)$ of Example 2

The set of feasible coalitions in this approach thus is given by

$$
\Phi_{D}^{c}:=\left\{S \subseteq N \mid P_{D}(i) \subset S \text { for all } i \in S\right\},
$$

which we refer to as the conjunctive feasible set of $D$.

Alternatively, in the disjunctive approach as developed in Gilles and Owen (1994) and van den Brink (1997), it is assumed that each player (except the top-players) needs permission from at least one of its predecessors before it is allowed to cooperate with other players. Consequently, a coalition is feasible if and only if every player in the coalition (except the top-players) has at least one predecessor who also belongs to the coalition. Thus, the feasible coalitions are the ones in the set

$$
\Phi_{D}^{d}:=\left\{S \subseteq N \mid P_{D}(i) \cap S \neq \emptyset \text { for all } i \in S \backslash T O P(D)\right\}
$$

which we refer to as the disjunctive feasible set of $D$.

Example 2 Consider the permission structure $(N, D)$ given by $N=\{1,2,3,4\}$ and $D=\{(1,2),(1,3),(2,4),(3,4)\}$, see Fig. 2. Then $\Phi_{D}^{c}=\{\{1\},\{1,2\},\{1,3\},\{1,2,3\}$, $\{1,2,3,4\}\}$ and $\Phi_{D}^{d}=\Phi_{D}^{c} \cup\{\{1,2,4\},\{1,3,4\}\}$.

An approach using restricted games similar to the approach of Myerson (1977) for communication graph games assigns to every coalition in a game with a permission structure the worth of its largest feasible subset. ${ }^{4}$ If the permission structure has a unique top-player and the (unrestricted) game is monotone (meaning that $v(S) \leq v(T)$ whenever $S \subseteq T \subseteq N$ ) then the conjunctive and disjunctive restricted games have at least one Core-stable payoff vector.

Why do these models capture the idea of a hierarchy? Since we are in the field of restricted cooperation, the answer must be found in the properties of the set of feasible coalitions. Algaba et al. (2004) show that the conjunctive and disjunctive feasible sets are antimatroids being well-known combinatorial structures that represent hierarchies, see Dilworth (1940) and Edelman and Jamison (1985). An antimatroid is defined by the following properties. A set of feasible coalitions $\mathcal{F} \subseteq 2^{N}$ satisfies accessibility if every nonempty feasible coalition has at least one player that can leave the coalition leaving behind a feasible subcoalition, i.e., $S \in \mathcal{F}, S \neq \emptyset$, implies that there exists an

\footnotetext{
${ }^{4}$ Different properties of communication feasible sets and conjunctive- or disjunctive feasible sets yield different properties of the restricted games. Whereas in communication graph games every coalition can be partitioned into feasible components, in a conjunctive- or disjunctive permission structure every coalition has a unique largest feasible subset.
} 
$i \in S$ such that $S \backslash\{i\} \in \mathcal{F}$. A set of feasible coalitions $\mathcal{F} \subseteq 2^{N}$ is closed under union if the union of any two feasible coalitions is also feasible, i.e., $S, T \in \mathcal{F}$ implies that $S \cup T \in \mathcal{F}$. Together with the empty set being feasible these two properties define an antimatroid. In addition, we require the set of feasible coalitions $\mathcal{F} \subseteq 2^{N}$ to be normal meaning that every player belongs to at least one feasible coalition, ${ }^{5}$ i.e., for every $i \in N$ there exists an $S \in \mathcal{F}$ such that $i \in S$.

Definition 1 A set of feasible coalitions $\mathcal{F} \subseteq 2^{N}$ is a normal antimatroid if it contains the empty set and satisfies normality, accessibility and is closed under union.

We refer to a player $i \in S \in \mathcal{F} \subseteq 2^{N}$ such that $S \backslash\{i\} \in \mathcal{F}$ as an extreme player of coalition $S$ in $\mathcal{F}$. By accessibility every nonempty feasible coalition in an antimatroid has at least one extreme player.

\section{Characterizing communication feasible sets}

To know whether one speaks about communication or hierarchies in restricted cooperation, one needs to know the properties of the set of feasible coalitions. In this section, we characterize the sets of feasible coalitions arising from communication restrictions and compare them with those of hierarchies represented by antimatroids.

Let $\mathcal{F} \subseteq 2^{N}$ be an arbitrary set of feasible coalitions. Since all singletons in a communication graph are connected, it follows that communication feasible sets arising from communication graphs contain the empty set and satisfy normality, i.e., every player belongs to at least one feasible coalition. Further, they also satisfy accessibility. They even satisfy the following stronger property. We say that a set of feasible coalitions $\mathcal{F} \subseteq 2^{N}$ satisfies 2-accessibility if every feasible coalition with two or more players has at least two players that can leave the coalition leaving behind a feasible coalition, i.e., $S \in \mathcal{F}$ with $|S| \geq 2$ implies that there exist $i, j \in S, i \neq j$, such that $S \backslash\{i\} \in \mathcal{F}$ and $S \backslash\{j\} \in \mathcal{F}$. Communication feasible sets are not closed under union (as is illustrated by the two connected coalitions $\{1,2\}$ and $\{5\}$ in Example 1 which union is not connected). However, as shown by Algaba et al. (2001), communication feasible sets satisfy the weaker union stability meaning that the union of two feasible coalitions that have a nonempty intersection is also feasible, i.e., $S, T \in \mathcal{F}$ with $S \cap T \neq \varnothing$ implies that $S \cup T \in \mathcal{F}$. It turns out that the four properties described above characterize the communication feasible sets.

Theorem 1 Let $\mathcal{F} \subseteq 2^{N}$ be a set of feasible coalitions. Then $\mathcal{F}$ is the communication feasible set of some communication graph if and only if $\mathcal{F}$ contains the empty set and satisfies normality, 2-accessibility, and union stability.

Proof (Only if) A communication feasible set containing the empty set and satisfying normality and union stability follows from Algaba et al. (2001). To show that it satisfies 2-accessibility, let $S \subseteq N$ with $|S| \geq 2$ be connected in communication graph $(N, G)$.

\footnotetext{
$\overline{5}$ For results on games on antimatroids, we refer to Algaba et al. $(2003,2004)$. For antimatroids that are not normal, similar results can be stated restricted to the class of players that belong to at least one feasible coalition.
} 
Note that for any pending player $i \in S$ it holds that $S \backslash\{i\}$ is connected, i.e., pending players are extreme players of $S$ in $\mathcal{F}_{G}$. We show 2-accessibility by induction on the number $|G(S)|$ of links between players in $S$. Since $S$ is connected in $(N, G)$ we have $|G(S)| \geq|S|-1$. If $|G(S)|=|S|-1$ then $(N, G(S))$ is cycle-free and thus has at least two pending players, which are extreme players of $S$ in $\mathcal{F}_{G}$. If $|G(S)|>|S|-1$, then there is a subgraph $G^{\prime} \subset G$ such that $S$ is connected in $\left(N, G^{\prime}\right)$ and $\left|G^{\prime}(S)\right|=|S|-1$. From above we know that $S$ has at least two extreme players in $\mathcal{F}_{G^{\prime}}$. Since $\mathcal{F}_{G^{\prime}} \subseteq \mathcal{F}_{G}$, every extreme player of $S$ in $\mathcal{F}_{G^{\prime}}$ is also an extreme player of $S$ in $\mathcal{F}_{G}$, and thus $S$ has at least two extreme players in $\mathcal{F}_{G}$.

(If) Suppose that $\mathcal{F} \subseteq 2^{N}$ satisfies the properties mentioned in the theorem. Consider the communication graph $G^{\mathcal{F}}=\{S \in \mathcal{F}|| S \mid=2\}$. It is sufficient to show that $\mathcal{F}$ is the set of connected coalitions in $\left(N, G^{\mathcal{F}}\right)$. By definition, $\mathcal{F}$ contains the empty set. Next we first show that all singletons are feasible. For $i \in N$, normality implies that there is an $S \in \mathcal{F}$ with $i \in S$. By 2-accessibility, it follows that there is a $j \in S \backslash\{i\}$ such that $S \backslash\{j\} \in \mathcal{F}$. Repeated application of 2-accessibility, each time deleting an extreme player different than $i$, eventually yields that $\{i\} \in \mathcal{F}$.

Finally, we show that for every $S \subseteq N$ with $|S| \geq 2$, it holds that $S \in \mathcal{F}$ if and only if $S$ is connected in $\left(N, G^{\mathcal{F}}\right)$.

("only if") Take $S \in \mathcal{F}$ with $|S| \geq 2$. We prove that $S$ is connected in $\left(N, G^{\mathcal{F}}\right)$ by induction on $|S|$. If $|S|=2$ then $S$ is connected in $\left(N, G^{\mathcal{F}}\right)$ by definition of $G^{\mathcal{F}}$. Proceeding by induction, suppose that $S^{\prime} \in \mathcal{F}$ is connected in $\left(N, G^{\mathcal{F}}\right)$ whenever $2 \leq\left|S^{\prime}\right|<|S|$. By 2 -accessibility there exist $i, j \in S$ such that $S \backslash\{i\}, S \backslash\{j\} \in \mathcal{F}$. The induction hypothesis implies that $S \backslash\{i\}$ and $S \backslash\{j\}$ are both connected in $\left(N, G^{\mathcal{F}}\right)$. Since $|S|>2$ there exists an $h \in S \backslash\{i, j\}$ such that there is a path from $i$ to $h$ and from $j$ to $h$ in $\left(N, G^{\mathcal{F}}\right)$ using only players from $S$. This implies that there is a path from $i$ to $j$ in $S$, and thus $S$ is connected in $\left(N, G^{\mathcal{F}}\right)$.

('if') Take $S \subseteq N$ connected in $\left(N, G^{\mathcal{F}}\right)$. We must prove that $S \in \mathcal{F}$. If $|S|=2$ then $S \in \mathcal{F}$ by definition of $G^{\mathcal{F}}$. If $|S|>2$ then $S$ is the union of all links in $S$, i.e., $S=\bigcup\left\{\{i, j\} \in G^{\mathcal{F}} \mid i, j \in S\right\}$. Since all these links $\{i, j\}$ belong to $\mathcal{F}$ by definition of $G^{\mathcal{F}}$ and $S$ is connected, union stability implies that $S \in \mathcal{F}$.

Usually the set of links $G$, being coalitions of size two, are considered as the basis of a communication network. Note that by applying 2-accessibility we can generate these bilateral links from any communication feasible set.

Comparing Theorem 1 with Definition 1, we conclude that communication feasible sets are characterized by similar properties that define hierarchical structures represented by normal antimatroids. To be specific, besides normality and feasibility of the empty set, both satisfy an accessibility and a union property. Obviously, 2-accessibility implies accessibility and thus communication feasible sets satisfy a stronger accessibility property. But since closedness under union implies union stability, antimatroids satisfy a stronger union property.

Also note that given 2-accessibility, normality implies that $\{i\} \in \mathcal{F}$ for all $i \in N$ as is the case for communication feasible sets. Given closedness under union, normality implies that $N \in \mathcal{F}$ as is the case for antimatroids. 


\section{Special classes of communication graphs}

In this section, we characterize some special classes of communication graphs that are often encountered in the economic literature. We do this by adding additional properties with respect to paths in a set of feasible coalitions. In the previous section, we showed that every coalition in a communication feasible set has at least two extreme players. We say that a coalition $S \in \mathcal{F} \subseteq 2^{N}$ is a path in $\mathcal{F}$ if it has exactly two extreme players. ${ }^{6}$ The path $S \in \mathcal{F}$ is called an $\{i, j\}$-path in $\mathcal{F}$ if it has $i$ and $j$ as extreme players. From now on it will be clear from the context whether we speak about a path $\left(i_{1}, \ldots, i_{k}\right)$ in a graph $(N, G)$ or a path $\left\{i_{1}, \ldots i_{k}\right\}$ in a set of feasible coalitions $\mathcal{F} \subseteq 2^{N}$

\subsection{Communication line-graphs}

A special class of communication networks that one encounters often in the economic literature are line-graphs, see, e.g., Greenberg and Weber (1986). For example, cooperative games arising from water distribution problems (see Ambec and Sprumont (2002)), polluted river games (see Ni and Wang (2007)), one-machine sequencing situations (see Curiel et al. (1994)), auction situations (see Graham et al. (1990)), and (dual) airport problems (see Littlechild and Owen (1973)) satisfy the property that the restricted game $v_{G}$ on an appropriately defined line-graph $G$ is equal to the unrestricted game $v$, see also van den Brink et al. (2007). Without loss of generality we consider line-graphs $G \subseteq G^{L}=\{\{i, i+1\} \mid i \in\{1, \ldots, n-1\}\}$, where $G^{L}$ is the full line-graph where each pair of consecutive players is directly linked. For convenience we denote coalition $\{i, i+1, \ldots, j-1, j\}$ by $[i, j]$. In van den Brink et al. (2007) it is shown that a sufficient condition for the existence of a Core-stable payoff vector for a restricted game on a line-graph is the game being linear-convex meaning that $v[i, j]-v[i+1, j]-v[i, j-1]+v[i+1, j-1] \geq 0$ for all connected coalitions $[i, j]]^{7}$

The examples mentioned above are linear-convex.

In the previous section, we characterized the communication feasible sets using union stability. Next we introduce a similar property with respect to paths. A set of feasible coalitions $\mathcal{F} \subseteq 2^{N}$ satisfies path union stability if the union of every pair of paths that have a nonempty intersection is also a path, i.e., if $S, T \in \mathcal{F}$ are both paths in $\mathcal{F}$ with $S \cap T \neq \emptyset$ then $S \cup T$ is a path in $\mathcal{F}$. Adding this property to those of Theorem 1 characterizes the communication feasible sets arising from communication line-graphs.

Theorem 2 Let $\mathcal{F} \subseteq 2^{N}$ be a set offeasible coalitions. Then $\mathcal{F}$ is the communication feasible set of some communication line-graph if and only if $\mathcal{F}$ contains the empty set and satisfies normality, 2-accessibility, union stability, and path union stability.

\footnotetext{
6 A path in $\mathcal{F}_{G}$ is also a path in the corresponding graph $(N, G)$.

7 Note that this is weaker than convexity of the game and neither stronger nor weaker than superadditivity. A game $v$ is convex if $v(S \cup T)+v(S \cap T) \geq v(S)+v(T)$ for all $S, T \subseteq N$.
} 
Proof (Only if) Let $(N, G)$ be a communication line-graph. $\mathcal{F}_{G}$ containing the empty set and satisfying normality, 2-accessibility, and union stability follows from Theorem 1 . The paths in a communication line-graph are exactly the connected (consecutive) coalitions $[i, j] . \mathcal{F}_{G}$ satisfying path union stability follows since $[i, j]$ and $[k, l]$ being two paths in $(N, G)$ with $j>k$ implies that their union $[i, l]$ is connected in $(N, G)$, and thus an $\{i, l\}$-path in $\mathcal{F}_{G}$.

(If) Suppose that $\mathcal{F} \subseteq 2^{N}$ satisfies the properties mentioned in the theorem. From Theorem 1, it follows that $\mathcal{F}$ is the communication feasible set of a communication graph $(N, G)$. Suppose that $(N, G)$ is not a line-graph. Then there is an $i \in N$ with $\left|R_{G}(i)\right| \geq 3$. If there exist distinct $h, j, k \in R_{G}(i)$ with $\{\{h, j\},\{h, k\},\{j, k\}\} \cap G=\emptyset$, then $\{i, h, j\}$ and $\{i, k\}$ are both paths in $\mathcal{F}$, but their union $\{i, j, h, k\}$ is not a path. Otherwise, there is a cycle between $i$ and two of its neighbors, say $\{h, j\} \in G$ for $h, j \in R_{G}(i)$. But then $\{i, j\}$ and $\{i, h\}$ are both paths in $\mathcal{F}$ but their union $\{i, j, h\}$ is not a path. In both cases we have a contradiction with path union stability of $\mathcal{F}$.

\subsection{Cycle-free communication graphs}

An important class of communication graphs that contains the communication linegraphs is that of cycle-free communication graphs as considered in, e.g., Le Breton et al. (1992) and Demange (1994, 2004). As mentioned before, they have shown that the Core of the restricted game $v_{G}$ is nonempty if the game is superadditive and the graph is cycle-free, respectively a tree. The communication feasible sets arising from cycle-free communication graphs that are not a line need not satisfy path union stability (as can seen from the cycle-free graph $(N, G)$ with $N=\{1, \ldots, 4\}$ and $G=\{\{1, j\} \mid j \in\{2,3,4\}\}$, where $\{1,2,3\}$ and $\{1,4\}$ are both paths in $\mathcal{F}_{G}$ but their union is not). However, requiring the path union stability condition only for the union of two paths that have a common extreme player, this property is satisfied for cyclefree communication graphs. We say that a set of feasible coalitions $\mathcal{F} \subseteq 2^{N}$ satisfies weak path union stability if the union of an $\{i, j\}$-path and an $\{i, k\}$-path, $j \neq k$, is also a path. Weakening path union stability in this way characterizes the communication feasible sets arising from cycle-free communication graphs.

Theorem 3 Let $\mathcal{F} \subseteq 2^{N}$ be a set offeasible coalitions. Then $\mathcal{F}$ is the communication feasible set of some cycle-free communication graph if and only if $\mathcal{F}$ contains the empty set and satisfies normality, 2-accessibility, union stability, and weak path union stability.

Proof (Only if) Let $(N, G)$ be a cycle-free communication graph. $\mathcal{F}_{G}$ containing the empty set and satisfying normality, 2-accessibility and union stability follows from Theorem 1. Note that for a cycle-free graph the paths in $\mathcal{F}_{G}$ exactly correspond to the paths in the graph $(N, G)$. Thus, $\mathcal{F}_{G}$ satisfying weak path union stability follows since $S$ being an $\{i, j\}$-path and $T$ being an $\{i, k\}$-path, $j \neq k$, implies that their union is a path from $j$ to $k$ in $(N, G)$, and thus $S \cup T$ is a $\{j, k\}$-path in $\mathcal{F}_{G}$.

(If) To prove the 'if' part, suppose that $\mathcal{F} \subseteq 2^{N}$ satisfies the properties mentioned in the theorem. We already showed in Theorem 1 that $\mathcal{F}$ is the communication feasible set of a communication graph $(N, G)$. Suppose that $(N, G)$ has a 
cycle. Then there exists a minimal cycle $\left(i_{1}, i_{2}, \ldots, i_{k}, i_{1}\right), k \geq 3$, such that there is no cycle $\left(h_{1}, h_{2}, \ldots, h_{g}, h_{1}\right)$ with $\left\{h_{1}, h_{2}, \ldots, h_{g}\right\} \subset\left\{i_{1}, i_{2}, \ldots, i_{k}\right\}$. Take any $l \in\{2, \ldots, k-1\}$. Then $\left\{i_{1}, \ldots, i_{l}\right\}$ is an $\left\{i_{1}, i_{l}\right\}$-path and $\left\{i_{l}, \ldots, i_{k}\right\}$ is an $\left\{i_{l}, i_{k}\right\}-$ path in $\mathcal{F}$, but their union is not a path, yielding a contradiction with weak path union stability of $\mathcal{F}$.

\subsection{Cycle-complete graphs}

For games on antimatroids, Algaba et al. (2004) show that adding to the properties of Definition 1 the requirement that every player belongs to exactly one coalition in which it is the only extreme player, characterizes the so-called poset antimatroids (which turn out to be exactly those antimatroids that can be the conjunctive feasible set of some acyclic permission structure). Since we showed that every connected coalition in a communication graph has at least two extreme players, we consider the following modification of this property. A set of feasible coalitions $\mathcal{F} \subseteq 2^{N}$ satisfies the path property if for every pair of players $i, j \in N$ there is at most one $\{i, j\}$-path.

Requiring this additional property for communication feasible sets exactly yields those that can arise from cycle-complete communication graphs. A communication graph is cycle-complete if, whenever there is a cycle, the subgraph restricted to the players in that cycle is complete, i.e., if there is a cycle $\left(i_{1}, \ldots, i_{k}, i_{1}\right)$ then $\{\{i, j\} \mid$ $\left.\{i, j\} \subseteq\left\{i_{1}, \ldots, i_{k}\right\}\right\} \subseteq G$. In van den Nouweland and Borm (1991) it is shown that the restricted game $v_{G}$ is convex, and thus its Core is nonempty, if the game is convex and the graph is cycle-complete. So, compared to the results of Le Breton et al. (1992) and Demange (1994, 2004) mentioned in the previous subsection, they show under a weaker condition on the communication graph (since cycle-completeness is implied by cycle-freeness) but stronger condition on the game (since convexity implies superadditivity) nonemptiness of the Core of the restricted game.

Theorem 4 Let $\mathcal{F} \subseteq 2^{N}$ be a set of feasible coalitions. Then $\mathcal{F}$ is the communication feasible set of some cycle-complete communication graph if and only if $\mathcal{F}$ contains the empty set and satisfies normality, 2-accessibility, union stability, and the path property.

Proof (Only if) Let $(N, G)$ be a cycle-complete communication graph. $\mathcal{F}_{G}$ containing the empty set and satisfying normality, 2-accessibility and union stability follows from Theorem 1. A path $\left(i_{1}, \ldots, i_{k}\right)$ from $i$ to $j$ in communication graph $(N, G)$ is a shortest path from $i$ to $j$ if there is no path $\left(h_{1}, \ldots, h_{g}\right)$ from $i$ to $j$ such that $\left\{h_{1}, \ldots, h_{g}\right\} \subset\left\{i_{1}, \ldots, i_{k}\right\}$. Since $(N, G)$ is cycle-complete, there is at most one shortest path between every pair of players. ${ }^{8}$. The shortest paths in a cycle-complete graph exactly correspond to the paths in $\mathcal{F}_{G}$. Therefore, for every $i, j \in N$ there is at most one $\{i, j\}$-path in $\mathcal{F}_{G}$.

\footnotetext{
${ }^{8}$ Let $H_{G}(i, j)=\left\{h \in N \mid\right.$ there is a path $\left(i_{1}, \ldots, i_{k}\right)$ from $i$ to $j$ in communication graph $(N, G)$ such that $\left.h \in\left\{i_{1}, \ldots, i_{k}\right\}\right\}$. If $\left(N, G\left(H_{G}(i, j)\right)\right)$ is cycle-free then there is at most one path between $i$ and $j$. (There is exactly one such a path if $i$ and $j$ are connected.) If $\left(H_{G}(i, j), G\left(H_{G}(i, j)\right)\right)$ is complete then $\{i, j\}$ is the unique shortest path. Otherwise, every path from $i$ to $j$ contains players from complete subgraphs such that there are only two players of this complete subgraph (one of them possibly being $i$ or $j$ ) that belong to the shortest path from $i$ to $j$.
} 
(If) Suppose that $\mathcal{F} \subseteq 2^{N}$ satisfies the properties mentioned in the theorem. We already showed in Theorem 1 that $\mathcal{F}$ is the communication feasible set of a communication graph $(N, G)$. Suppose that $(N, G)$ is not cycle-complete. Then there is a cycle $\left(i_{1}, i_{2}, \ldots, i_{k}, i_{1}\right), k \geq 4$, with $i_{m} \in\left\{i_{3}, \ldots, i_{k-1}\right\}$ such that $R_{G}\left(i_{1}\right) \cap$ $\left\{i_{1}, i_{2}, \ldots, i_{k}\right\}=\left\{i_{2}, i_{k}\right\}$ and $R_{G}\left(i_{m}\right) \cap\left\{i_{1}, i_{2}, \ldots, i_{k}\right\}=\left\{i_{m-1}, i_{m+1}\right\}$. (Note that a cycle of length 3 is complete.) Take any $l \in\{3, \ldots, k-1\}$. Then $\left\{i_{1}, \ldots, i_{l}\right\}$ and $\left\{i_{l}, \ldots, i_{k}, i_{1}\right\}$ are both $\left\{i_{1}, i_{l}\right\}$-paths in $\mathcal{F}$, yielding a contradiction with the path property.

The reason why the path property still allows complete subgraphs is that in a cycle of length 3 (i.e., a cycle of three players) each pair of players in this cycle is the unique path of this pair of players in the corresponding communication feasible set. Therefore, in the previous subsections on cycle-free graphs we needed (weak) path union stability to exclude cycles of length 3 .

Alternatively, it can be shown that communication feasible sets of cycle-complete communication graphs are characterized by replacing the path property in Theorem 4 by closedness under intersection meaning that the intersection of any two feasible coalitions is also feasible, i.e., $S, T \in \mathcal{F}$ implies that $S \cap T \in \mathcal{F}$.

\subsection{Connectedness}

In the communication graphs considered in this section, we allowed the graph to be not connected. In many applications, connectedness of the graph is required, see, e.g., Demange $(1994,2004)$ who considers trees. We can easily adapt the characterizations given in the previous sections by adding connectedness for sets of feasible coalitions. A set of feasible coalitions $\mathcal{F} \subseteq 2^{N}$ is connected if for every $i, j \in N$ there is an $S \in \mathcal{F}$ with $\{i, j\} \subseteq S$. We state this result for trees, but similarly we can characterize connected line-graphs and cycle-complete graphs.

Theorem 5 Let $\mathcal{F} \subseteq 2^{N}$ be a set of feasible coalitions. Then $\mathcal{F}$ is the communication feasible set of some communication tree if and only if $\mathcal{F}$ contains the empty set and satisfies normality, 2-accessibility, union stability, weak path union stability, and connectedness.

Proof (Only if) Let $(N, G)$ be a communication tree. $\mathcal{F}_{G}$ containing the empty set and satisfying normality, 2-accessibility, union stability, and weak path union stability follows from Theorem 3. $\mathcal{F}_{G}$ satisfying connectedness follows since $N \in \mathcal{F}_{G}$ if $(N, G)$ is a tree.

(If) To prove the 'if' part, suppose that $\mathcal{F} \subseteq 2^{N}$ satisfies the properties mentioned in the theorem. From Theorem 3, it follows that $\mathcal{F}$ is the communication feasible set of a cycle-free communication graph $(N, G)$. Suppose that $(N, G)$ is not connected. Then there are at least two components $T^{1}, T^{2}$ in $(N, G)$. Taking $i \in T^{1}$ and $j \in T^{2}$, every $S \subseteq N$ with $\{i, j\} \subseteq S$ is not feasible, yielding a contradiction with $\mathcal{F}=\mathcal{F}_{G}$ being connected. 


\section{Concluding remarks}

In this article, we characterized the communication feasible sets that can be the set of connected coalitions in a communication graph network and compared the characterizing properties to those of a hierarchical structure represented by an antimatroid (including permission structures). We showed that these sets differ with respect to the accessibility and union property they satisfy, where communication feasible sets satisfy a stronger accessibility property, while hierarchies (i.e., antimatroids) satisfy a stronger union property. We also characterized communication feasible sets of special subclasses of communication networks, in particular, line, cycle-free, tree, and cycle-complete graphs. From the literature, sufficient conditions for the existence of Core-stable payoff vectors on these communication structures can be found which we summarize in Table 1 (where the second column says which properties additional to those of Theorem 1 characterize that structure and the third column gives sufficient conditions on the game to have a nonempty Core given a communication graph with those properties).

Demange (2004) considers restricted cooperation in a model where the players in a TU-game belong to a hierarchy represented by a rooted or directed tree. In that article, the feasible coalitions (or teams) in this hierarchy are those coalitions $S \subseteq N$ such that for every pair of players $i, j \in S$ either (i) there is a directed path from $i$ to $j$, or (ii) there is a directed path from $j$ to $i$, or (iii) there is another player $h \in S \backslash\{i, j\}$ such that there is a directed path from $h$ to $i$ and from $h$ to $j$. But this implies that the feasible coalitions are exactly the connected coalitions in the underlying undirected communication graph $\left(N, G^{D}\right)$ with $G^{D}=\{\{i, j\} \subseteq N \mid i \neq j$ and $\{(i, j),(j, i)\} \cap D \neq \emptyset\}$, where the communication graph is a tree. Equivalently, we now can verify that the corresponding sets of feasible coalitions satisfy the properties of Theorem 5 .

We conclude that characterizations of sets of feasible coalitions arising from hierarchies and communication networks is important to build a consistent theory of such networks and compare them in economic and political organizations. Combining game theory with studying relational structures by looking at properties of their sets of feasible coalitions provides useful tools for this purpose. We agree with Chwe (2000) that although "collective action depends on both social structure and individual incentives, these integral aspects have been formalized separately, in the fields of social network theory and game theory." Whereas Chwe (2000) considers these integral aspects together in a noncooperative model, in this article, we took a step to consider communication and hierarchy together in a cooperative framework.

Table 1 Properties on networks and games

\begin{tabular}{lll}
\hline & Characterizing properties & Properties on $v$ \\
\hline Line & Path union stability & Linear-convexity \\
Cycle-free & Weak path union stability & Superadditivity \\
Cycle-complete & Path property & Convexity \\
Tree & Weak path union stability, & Superadditivity \\
& connectedness & \\
\hline
\end{tabular}


Open Access This article is distributed under the terms of the Creative Commons Attribution Noncommercial License which permits any noncommercial use, distribution, and reproduction in any medium, provided the original author(s) and source are credited.

\section{References}

Aghion P, Tirole J (1997) Formal and real authority in organizations. J Political Econ 105:1-29

Algaba E, Bilbao JM, Borm P, López JJ (2001) The Myerson value for union stable structure. Math Methods Oper Res 54:359-371

Algaba E, Bilbao JM, van den Brink R, Jiménez-Losada A (2003) Axiomatizations of the Shapley value for cooperative games on antimatroids. Math Methods Oper Res 57:49-65

Algaba E, Bilbao JM, van den Brink R, Jiménez-Losada A (2004) Cooperative games on antimatroids. Discret Math 282:1-15

Ambec S, Sprumont Y (2002) Sharing a river. J Econ Theory 107:453-462

Bala V, Goyal S (2000) A noncooperative model of network formation. Econometrica 68:1181-1229

Chwe MS-Y (2000) Communication and coordination in social networks. Rev Econ Stud 67:1-16

Curiel I, Potters J, Rajendra Prasad V, Tijs S, Veltman B (1994) Sequencing and cooperation. Oper Res 54:323-334

Demange G (1994) Intermediate preferences and stable coalition structures. J Math Econ 23:45-58

Demange G (2004) On group stability in hierarchies and networks. J Political Econ 112:754-778

Dewatripont M, Tirole J (2005) Modes of communication. J Political Econ 113:1217-1238

Dilworth RP (1940) Lattices with unique irreducible decompositions. Ann Math 41:771-777

Edelman PH, Jamison RE (1985) The theory of convex geometries. Geom Dedic 19:247-270

Gilles RP, Owen G (1994) Games with permission structures: the disjunctive approach. Mimeo, Department of Economics, Virginia Polytechnic Institute and State University, Blacksburg, Virginia

Gilles RP, Owen G, van den Brink R (1992) Games with permission structures: the conjunctive approach. Int J Game Theory 20:277-293

Graham DA, Marshall RC, Richard J-F (1990) Differential payments within a bidder coalition and the Shapley value. American Econ Rev 80:493-510

Greenberg J, Weber S (1986) Strong Tiebout equilibrium under restricted preferences domain. J Econ Theory $38: 101-117$

Hart O, Moore J (2005) On the design of hierarchies: coordination versus specialization. J Political Econ 113:675-702

Jackson MO, Wolinsky A (1996) A strategic model of social and economic networks. J Econ Theory 71:4474

Kaneko M, Wooders M (1982) Cores of partitioning games. Math Soc Sci 3:313-327

Le Breton G, Owen G, Weber S (1992) Strongly balanced cooperative games. Int J Game Theory 20: 419-427

Littlechild SC, Owen G (1973) A simple expression for the Shapley value in a special case. Manag Sci 20:370-372

Myerson RB (1977) Graphs and cooperation in games. Math Oper Res 2:225-229

Ni D, Wang Y (2007) Sharing a polluted river. Games Econ Behav 60:176-186

Shapley LS (1953) A value for $n$-person games. In: Kuhn HW, Tucker AW (eds) Annals of Mathematics Studies, vol 28 (Contributions to the Theory of Games, vol 2). Princeton University Press, Princeton pp 307-317

van den Brink R (1997) An axiomatization of the disjunctive permission value for games with a permission structure. Int J Game Theory 26:27-43

van den Brink R, Gilles RP (1996) Axiomatizations of the conjunctive permission value for games with permission structures. Games Econ Behav 12:113-126

van den Brink R, van der Laan G, Vasil'ev V (2007) Component efficient solutions in line-graph games with applications. Component efficient Solutions inline-graph games with applications. Econ Theory 33:349-364

van den Nouweland A, Borm P (1991) On the convexity of communication games. Int J Game Theory 19:421-430 